\title{
Some Chemical Aspects of Human Brain Development. II. Phosphoglyceride Fatty Acids
}

\author{
Manuela Martinez, Carmen Conde, and Angel Ballabriga ${ }^{[31]}$ \\ Autonomous University, School of Medicine, Children's Hospital of the "Seguridad Social," Barcelona, Spain
}

\begin{abstract}
Extract
The fatty acids of total phosphoglycerides (TPG), ethanolamine phosphoglycerides (EPG), and choline phosphoglycerides (CPG) were obtained by mild alkaline transmethylation from lipid extracts of whole cerebrum and then analyzed by gas chromatography. A complete brain hemisphere from each of the 34 newborn infants reported previously was homogenized and its lipids extracted according to the procedure specified in that report. As the gestational age of the children went up, a statistically significant increase of the $n-3 / n-6$ ratio and, especially, of the $22: 4(n-6) / 22: 5(n-6)$ index was observed. Other ratios, such as the $n-6 / n-9$ and the $18: 0 / 18: 1(n-9)$, were also studied in the fatty acid patterns of EPG and CPG. Both of them showed significant increases with the gestational age of the infants. The $[22: 4(\mathrm{n}-6)+22: 5(\mathrm{n}-6)] /$ $20: 4(n-6)$ index, an indicator of the elongation process of arachidonic acid, on the other hand, did not show appreciable changes with maturation in TPG during this period of life. When ethanolamine and choline phosphoglycerides were analyzed separately, however, the elongation of arachidonic acid did rise with the gestational age in the former whereas it decreased in the latter.
\end{abstract}

\section{Speculation}

As intrauterine maturation of the human brain progresses, changes in the polyunsaturated fatty acid patterns, contrary to those observed in undernourished animals, take place. Among these changes, the increase of the $n-3$ family fatty acids and the decrease of the 22:5(n-6), reported in humans during the entire life span, can already be found in such early stages of life as selected for this study and within so narrow a range of gestational ages.

\section{Introduction}

Among the phosphoglycerides, only phosphatidal ethanolamine (ethanolamine plasmalogen) and triphosphoinositide are characteristically found in myelin. Phosphatidyl ethanolamine and phosphatidyl choline, on the other hand, are considered general components of cellular membranes and subcellular particles. As the relative proportion of the different brain phos- pholipids changes with age and, in the earliest stages of life, choline and ethanolamine phosphoglycerides are dominant, the study of total phosphoglyceride fatty acids in children is not directly related to myelination, but represents brain cellular maturation in a more general way. Some investigators $[1,25]$, however, have related the polyunsaturated fatty acids, characteristic of phosphoglycerides, to myelin lipids in an indirect way by suggesting that a deficiency in essential 
fatty acids would affect the production of myelin lipids.

A fair number of studies on essential fatty acids have been carried out in animals, where the fatty acid composition of brain and other organs can be related to fat intake. Successive investigations $[2,10-12,16,17$, 20-22, 27] showed a competitive inhibition between fatty acids of the linolenate (n-3, [29]) and linoleate (n6) series, as well as between the $n-9$ (oleate) and $n-6$ families $[5,8,9,15-17,25]$.

There is a limited number of studies on the fatty acid composition of the human brain. O'Brien et al. [18] and O'Brien and Sampson [19] determined several brain lipids and their fatty acid and fatty aldehyde composition in small groups of humans. They found marked differences in gray and white matter, but practically no age-dependent changes.

A detailed study carried out by Svennerholm [24] in a group of 11 human brains within a very wide range of ages (from a fetus to an 82-year-old adult) showed that the fatty acid patterns of the various phosphoglycerides in gray and white matter change throughout the whole life span. As far as the polyunsaturated fatty acids are concerned, he found that those of the $n-6$ series predominated in the youngest brains, especially in EPG of gray matter, whereas those of the n-3 family increased correpondingly with age [30].

White et al. [28] analyzed the EPG fatty acids in a series of nine human brains, also ranging widely in age (from fetal age to 77 years). Between fetal and adolescent ages they found a decrease of 20:4(n-6) and monoenes (18:1(n-9) and 20:1(n-9)). They did not notice any significant changes in the 22:6(n-3) percentage with aging.

In the present report, the phosphoglyceride fatty acids of the whole cerebrum were studied in a homogeneous group of 34 newborn infants of different gestational ages in order to ascertain whether the changes described by other workers throughout the entire life span could already be detected during the intrauterine period. In fact, none of the infants had yet been fed and they had died within the first hours of life. Furthermore, their mothers belonged to a well nourished population. So, the changes in the fatty acid patterns, if present, would indicate a spontaneous process of brain maturation as the gestation progressed.

\section{Material and Methods}

The human material consisted of the 34 newborn brains specified in the first part of this study [3]. It must be emphasized that a very strict criterion was followed in the selection of the cases, rigorously excluding all the children whose clinical examination suggested any degree of brain damage or whose autopsy showed cerebral hemorrhage or other brain alterations.

The lipid extracts were obtained by the procedure mentioned in that paper. The lower phase of these extracts was employed to analyze the fatty acid composition of TPG, EPG, and CPG. The isolation of the main lipids was accomplished by thin layer chromatography. The solvent systems were petroleum ether-ethyl ether-acetic acid, 90/10/1, for total phospholipids, and chloroform-methanol-water $65 / 25 / 4$ for ethanolamine and choline phosphoglycerides. No distinction was made between phosphatidyl ethanolamine and phosphatidal ethanolamine, both present in the EPG spot. The spots were scraped off and transferred into special glass-stoppered tubes. After drying overnight in a vacuum dessicator filled with silica gel, $2 \mathrm{ml} 0.1 \mathrm{~N}$ sodium methoxide in dry methanol were added and the tubes stoppered and shaken for $1 \mathrm{hr}$, according to Svennerholm's method [24]. After acidification with $0.1 \mathrm{~N}$ acetic acid, the fatty acids from each spot were extracted three times with petroleum ether, washed twice with water, and dried with anhydrous sodium sulfate. This method of mild alkaline methanolysis was chosen because it is very efficient only for glycerolipids and it does not attack the double bonds. It was particularly useful in the present study because, as the sphingomyelin were unattacked by sodium methoxide-methanol at room temperature, only the total phosphoglyceride fatty acids were liberated from the phospholipid spot; no further method of separation was required.

After evaporation to a final volume of 10-20 $\mu$, the samples were injected into the gas chromatograph. A Perkin-Elmer model 900 gas chromatograph was used, equipped with flame ionization detectors. The separation was performed on a 10 foot long, $1 / 8$ inch $O D$ stainless steel column, packed with $15 \%$ ethylene glycol succinate (LAC-4R 886) on 80-100 mesh Varaport 30 , programming from $160^{\circ}-200^{\circ}$, at a rate of $2^{\circ} / \mathrm{min}$. The peak areas were measured by triangulation and the results expressed as percentage of total area, which corresponded very closely to weight percentage, because of the linear response of the flame ionization detectors.

The statistical analyses were carried out with the help of an Olivetti programmer, model P203, and a Hewlett-Packard model $9100 \mathrm{~A}$ electronic calculator. The plotting and drawing of curvilinear regression 
lines was performed with a Hewlett-Packard plotter, model $9125 \mathrm{~A}$.

\section{Results and Discussion}

When the different fatty acids of TPG were compared in the whole series of children (Tables I and II), a definite increase of the $\mathrm{n}-3$ family fatty acids, mainly of the 22:6(n-3), was found as the gestational age of the infants went up $(P<0.01)$. This rise of the $\mathrm{n}-3$ fatty acids with maturation was in agreement with Svennerholm's findings during the entire postnatal life [24], in spite of our extremely narrow range of ages. The sum of all the $n-6$ fatty acids, on the other hand, did not vary during this period of life.

Nevertheless, the overall changes do not represent accurately what is happening with the fatty acids of different families in each individual case. So, in order to study these variations in a statistically valid way, the sum of the $\mathrm{n}-3$ series fatty acids was divided by the sum of those of the $n-6$ series, case by case, and the resultant coefficient was plotted against the gestational age of the children. In TPG it was found that there was no increase of the $n-3 / n-6$ ratio until the 38 th week of gestational age, the youngest children giving the greatest dispersion of values. From the 38 th week onwards, the ratio showed a clear rise, although the few cases in this period did not allow drawing statistical conclusions.

There was also found a very pronounced variation of the relative proportion between the two $n-6$ fatty acids with 22 carbon atoms: the lower the gestational age of the infants, the higher the 22:5(n-6), while its precursor, 22:4(n-6), moved in the opposite direction:

Table I. Fatty acid composition of total phosphoglycerides in newborn human brain (22-29 weeks gestational age $)^{1}$

\begin{tabular}{|c|c|c|c|c|c|c|c|c|c|c|c|c|c|c|c|c|c|}
\hline & $\begin{array}{c}\text { Case } 35 \\
\quad \text { (F, } \\
22 \mathrm{wk}, \\
920 \mathrm{~g})\end{array}$ & $\begin{array}{c}\text { Case 6 } \\
\text { (M, } \\
23 \mathrm{wk}, \\
590 \mathrm{~g})\end{array}$ & $\begin{array}{c}\text { Case } 32 \\
(\mathrm{~F}, \\
23 \mathrm{wk}, \\
1,240 \mathrm{~g})\end{array}$ & $\begin{array}{c}\text { Case } 31 \\
(\mathrm{~F}, \\
24 \mathrm{wk}, \\
700 \mathrm{~g})\end{array}$ & $\begin{array}{c}\text { Case } 36 \\
(\mathrm{~F}, \\
24 \mathrm{wk}, \\
800 \mathrm{~g})\end{array}$ & $\begin{array}{c}\text { Case } 38 \\
(\mathrm{~F}, \\
24 \mathrm{wk}, \\
1,060 \mathrm{~g})\end{array}$ & $\begin{array}{c}\text { Case } 27 \\
(\mathrm{M}, \\
24 \mathrm{wk}, \\
1,100 \mathrm{~g})\end{array}$ & $\begin{array}{c}\text { Case } 24 \\
(\mathrm{M}, \\
25 \mathrm{wk} \\
850 \mathrm{~g})\end{array}$ & $\begin{array}{c}\text { Case 11 } \\
(\mathrm{M}, \\
26 \mathrm{wk}, \\
620 \mathrm{~g})\end{array}$ & $\begin{array}{c}\text { Cass } 42 \\
\text { (M, } \\
26 \mathrm{wk}, \\
850 \mathrm{~g})\end{array}$ & $\begin{array}{l}\text { Case } 14 \\
\text { (M, } \\
26 \mathrm{wk}, \\
790 \mathrm{~g})\end{array}$ & $\begin{array}{c}\text { Case } 29 \\
(\mathrm{M}, \\
26 \mathrm{wk}, \\
900 \mathrm{~g})\end{array}$ & $\begin{array}{c}\text { Case } 18 \\
(\mathrm{M}, \\
26 \mathrm{wk} \\
1,000 \mathrm{~g})\end{array}$ & $\begin{array}{c}\text { Case } 25 \\
(\mathrm{~F}, \\
27 \mathrm{wk} \\
1,350 \mathrm{~g})\end{array}$ & $\begin{array}{c}\text { Case } 20 \\
(\mathrm{~F}, \\
28 \mathrm{wk}, \\
1,000 \mathrm{~g})\end{array}$ & $\begin{array}{c}\text { Case } 40 \\
(\mathrm{M}, \\
29 \mathrm{wk} \\
850 \mathrm{~g})\end{array}$ & $\begin{array}{c}\text { Case 23 } \\
\quad(\mathrm{F}, \\
29 \mathrm{wk}_{3} \\
1,100 \mathrm{~g})\end{array}$ \\
\hline $14: 0$ & 2.00 & 2.82 & 2.05 & 2.34 & 2.11 & 2.22 & 2.70 & 2.26 & 2.57 & 2.49 & 2.84 & 2.18 & 2.93 & 2.15 & 2.02 & 2.18 & 2.10 \\
\hline $16: 0$ & 28.62 & 34.23 & 29.20 & 29.85 & 30.03 & 29.02 & 37.46 & 29.52 & 30.00 & 29.73 & 35.93 & 28.68 & 33.57 & 30.25 & 29.12 & 28.74 & 31.26 \\
\hline $16: 1(n-7)$ & 3.98 & 4.23 & 3.90 & 4.65 & 4.23 & 4.70 & 4.56 & 4.48 & 4.37 & 3.94 & 4.35 & 4.73 & 4.35 & 4.13 & 4.13 & 4.20 & 4.06 \\
\hline $18: 0$ & 18.54 & 19.82 & 18.15 & 17.43 & 17.88 & 18.10 & 21.85 & 17.97 & 17.78 & 17.96 & 21.23 & 18.00 & 20.06 & 17.75 & 17.88 & 18.23 & 18.50 \\
\hline $18: 1(n-9)$ & 15.80 & 18.40 & 15.60 & 16.54 & 15.58 & 15.90 & 17.96 & 16.25 & 16.38 & 16.53 & 18.32 & 16.38 & 17.83 & 15.76 & 15.55 & 15.74 & 16.03 \\
\hline $18: 2(n-6)$ & 0.45 & 0.21 & 0.38 & 0.48 & 0.70 & 0.50 & 0.20 & 0.45 & 0.46 & 0.32 & 0.37 & 0.38 & 0.62 & 0.35 & 0.37 & 0.42 & 0.37 \\
\hline $\begin{array}{c}18: 3(n-3)+ \\
20: 1(n-9)\end{array}$ & 0.50 & 0.63 & 0.53 & 0.58 & 0.53 & 0.50 & 0.50 & 0.53 & 0.59 & 0.53 & 0.60 & 0.60 & 0.58 & 0.53 & 0.52 & 0.45 & 0.45 \\
\hline $20: 3(n-9)$ & 0.44 & 0.70 & 0.54 & 0.66 & 0.41 & 0.71 & 0.44 & 0.41 & 0.50 & 0.40 & 0.42 & 0.84 & 0.38 & 0.50 & 0.42 & 0.40 & 0.52 \\
\hline $20: 3(n-6)$ & 0.58 & 0.40 & 0.57 & 0.56 & 0.66 & 0.70 & 0.32 & 0.61 & 0.62 & 0.45 & 0.46 & 0.67 & 0.46 & 0.60 & 0.50 & 0.54 & 0.51 \\
\hline $20: 4(n-6)$ & 10.74 & 7.20 & 10.10 & 10.66 & 10.90 & 10.70 & 5.40 & 10.47 & 10.79 & 10.54 & 6.38 & 10.27 & 7.57 & 10.10 & 10.52 & 10.98 & 9.22 \\
\hline $22: 4(n-6)$ & 5.86 & 4.07 & 5.82 & 5.24 & 5.74 & 5.53 & 3.20 & 5.70 & 5.24 & 6.16 & 3.35 & 5.28 & 4.81 & 5.73 & 6.04 & 6.44 & 5.13 \\
\hline $22: 5(n-6)$ & 3.49 & 2.12 & 3.36 & 3.35 & 4.04 & 3.60 & 1.75 & 3.40 & 3.49 & 3.29 & 1.71 & 4.15 & 2.53 & 3.48 & 3.59 & 4.12 & 3.05 \\
\hline $22: 5(n-3)$ & 0.28 & 0.20 & 0.24 & 0.22 & 0.23 & 0.26 & 0.14 & 0.25 & 0.21 & 0.28 & 0.16 & 0.24 & 0.14 & 0.20 & 0.28 & 0.22 & 0.22 \\
\hline $22: 6(n-3)$ & 8.72 & 4.97 & 9.56 & 7.44 & 6.96 & 7.56 & 3.52 & 7.70 & 7.00 & 7.38 & 3.88 & 7.60 & 4.17 & 8.47 & 9.06 & 7.34 & 8.58 \\
\hline
\end{tabular}

$1 \mathrm{~F}$ : female; M: male.

Table II. Fatty acid composition of total phosphoglycerides in newborn human brain (31-45 weeks gestational age $)^{1}$

\begin{tabular}{|c|c|c|c|c|c|c|c|c|c|c|c|c|c|c|c|c|c|}
\hline & $\begin{array}{c}\text { Case } 22 \\
(\mathrm{~F}, \\
31 \mathrm{wk}, \\
1,300 \mathrm{~g})\end{array}$ & $\begin{array}{c}\text { Case } 41 \\
(\mathrm{~F}, \\
32 \mathrm{wk}, \\
1,560 \mathrm{~g})\end{array}$ & $\begin{array}{c}\text { Case } 19 \\
\text { (F, } \\
32 \mathrm{wk}, \\
2,000 \mathrm{~g})\end{array}$ & $\begin{array}{c}\text { Case } 4 \\
(\mathrm{~F}, \\
33 \mathrm{wk}, \\
1,700 \mathrm{~g})\end{array}$ & $\begin{array}{c}\text { Case } 28 \\
(\mathrm{~F}, \\
33 \mathrm{wk}, \\
3,030 \mathrm{~g})\end{array}$ & $\begin{array}{c}\text { Case } 13 \\
(\mathrm{~F}, \\
35 \mathrm{wk}, \\
2,300 \mathrm{~g})\end{array}$ & $\begin{array}{c}\text { Case } 3 \\
(\mathrm{M}, \\
35 \mathrm{wk}, \\
3,500 \mathrm{~g})\end{array}$ & $\begin{array}{c}\text { Case } 34 \\
(\mathrm{~F}, \\
36 \mathrm{wk}, \\
2,300 \mathrm{~g})\end{array}$ & $\begin{array}{c}\text { Case } 9 \\
(\mathrm{M}, \\
37 \mathrm{wk}, \\
2,550 \mathrm{~g})\end{array}$ & $\begin{array}{c}\text { Case } 16 \\
(\mathrm{M}, \\
38 \mathrm{wk}, \\
2,850 \mathrm{~g})\end{array}$ & $\begin{array}{c}\text { Case } 7 \\
(\mathrm{M}, \\
39 \mathrm{wk} \\
2,970 \mathrm{~g})\end{array}$ & $\begin{array}{c}\text { Case } 10 \\
(\mathrm{M}, \\
40 \mathrm{wk} \\
3,400 \mathrm{~g})\end{array}$ & $\begin{array}{c}\text { Case } 30 \\
(\mathrm{M}, \\
42 \mathrm{wk} \\
3,025 \mathrm{~g})\end{array}$ & $\begin{array}{c}\text { Case } 33 \\
(\mathrm{~F}, \\
42 \mathrm{wk}, \\
4,140 \mathrm{~g})\end{array}$ & $\begin{array}{c}\text { Case } 8 \\
(\mathrm{M}, \\
43 \mathrm{wk}, \\
3,500 \mathrm{~g})\end{array}$ & $\begin{array}{c}\text { Case } 5 \\
(\mathrm{~F}, \\
44 \mathrm{wk}, \\
4,100 \mathrm{~g})\end{array}$ & $\begin{array}{c}\text { Case } 21 \\
\text { (F, } \\
45 \mathrm{wk}, \\
3,500 \mathrm{~g})\end{array}$ \\
\hline $14: 0$ & 2.03 & 1.80 & 1.37 & 2.31 & 1.86 & 2.12 & 1.84 & 2.00 & 1.58 & 2.21 & 1.64 & 1.71 & 1.46 & 1.67 & 1.44 & 1.34 & 1.25 \\
\hline $16: 0$ & 28.67 & 28.74 & 30.48 & 33.81 & 29.30 & 32.31 & 29.17 & 33.84 & 28.36 & 31.46 & 28.35 & 32.33 & 29.88 & 33.40 & 28.90 & 29.07 & 28.53 \\
\hline $16: 1(n-7)$ & 3.96 & 4.50 & 3.81 & 4.50 & 4.35 & 3.52 & 3.35 & 4.43 & 3.62 & 4.04 & 3.86 & 4.09 & 3.53 & 4.15 & 3.06 & 3.24 & 4.22 \\
\hline $18: 0$ & 18.78 & 18.44 & 18.77 & 20.55 & 17.98 & 20.54 & 19.74 & 18.60 & 19.40 & 20.75 & 20.02 & 21.26 & 19.30 & 19.96 & 19.94 & 21.76 & 18.98 \\
\hline $18: 1(n-9)$ & 15.60 & 16.54 & 15.20 & 17.16 & 15.81 & 16.65 & 15.07 & 17.80 & 15.13 & 15.98 & 15.19 & 15.84 & 14.92 & 17.50 & 15.17 & 15.83 & 15.76 \\
\hline $18: 2(n-6)$ & 0.40 & 0.27 & 0.47 & 0.28 & 0.36 & 0.39 & 0.40 & 0.34 & 0.37 & 0.62 & 0.33 & 0.29 & 0.35 & 0.27 & 0.30 & 0.28 & 0.38 \\
\hline $\begin{array}{c}18: 3(\mathrm{n}-3)+ \\
20: 1(\mathrm{n}-9)\end{array}$ & 0.46 & 0.30 & 0.39 & 0.48 & 0.52 & 0.46 & 0.41 & 0.45 & 0.40 & 0.42 & 0.38 & 0.35 & 0.38 & 0.51 & 0.32 & 0.22 & 0.42 \\
\hline $20: 3(n-9)$ & 0.38 & 0.68 & 0.39 & 0.38 & 0.82 & 0.27 & 0.30 & 0.40 & 0.43 & 0.22 & 0.43 & 0.36 & 0.31 & 0.41 & 0.29 & 0.55 & 0.49 \\
\hline $20: 3(n-6)$ & 0.55 & 0.50 & 0.73 & 0.52 & 0.83 & 0.73 & 0.86 & 0.62 & 0.70 & 0.72 & 0.75 & 0.57 & 0.97 & 0.70 & 0.83 & 0.96 & 1.00 \\
\hline $20: 4(n-6)$ & 10.70 & 9.96 & 9.50 & 7.90 & 11.00 & 8.32 & 10.21 & 8.00 & 10.53 & 8.54 & 9.65 & 7.97 & 10.07 & 7.54 & 9.97 & 9.17 & 9.48 \\
\hline $22: 4(n-6)$ & 6.20 & 6.46 & 6.70 & 4.31 & 6.12 & 5.55 & 6.76 & 4.98 & 7.00 & 5.93 & 6.39 & 5.38 & 6.38 & 4.68 & 6.90 & 5.67 & 5.85 \\
\hline $22: 5(n-6)$ & 3.70 & 3.26 & 3.20 & 2.16 & 3.58 & 2.50 & 3.24 & 2.33 & 3.53 & 2.68 & 3.38 & 2.61 & 2.49 & 1.75 & 2.49 & 1.94 & 1.89 \\
\hline $22: 5(\mathrm{n}-3)$ & 0.24 & 0.23 & 0.19 & 0.12 & 0.17 & 0.24 & 0.21 & 0.16 & 0.20 & 0.24 & 0.22 & 0.30 & 0.33 & 0.25 & 0.32 & 0.34 & 0.53 \\
\hline $22: 6(\mathrm{n}-3)$ & 8.33 & 8.32 & 8.80 & 5.52 & 7.30 & 6.40 & 8.44 & 6.05 & 8.75 & 6.19 & 9.41 & 6.94 & 9.63 & 7.21 & 10.07 & 9.63 & 11.22 \\
\hline
\end{tabular}

$1 \mathrm{~F}$ : feraale; $\mathrm{M}$ : male. 
the lower the gestational age, the lower also the $22: 4(n-6)$.

The reciprocal variations of these two fatty acids have already been reported by other workers [20, 27] in whose studies animals were fed diets deficient in essential fatty acids. The passing from 22:4(n-6) to $22: 5(n-6)$ is a desaturation process. Therefore, the higher the $22: 5(n-6)$ the more active the desaturation of the $22: 4(n-6)$ will be. Inversely, when the $22: 5(n-6)$ is low it indicates that its precursor, 22:4(n-6), is being desaturated very little. As a result of this, a product of unsaturation to precursor ratio $(22: 5(\mathrm{n}-6) / 22: 4(\mathrm{n}-6))$ would indicate what the $22: 4(\mathrm{n}-6)$ desaturation is like, in the sense that a high ratio would mean a very active desaturation, and vice versa (Paoletti and co-workers $[7,20])$. In the present study, however, the opposite ratio was chosen so that all the indices increased with the gestational age and could be compared more easily. Thus, the $22: 4(\mathrm{n}-6) / 22: 5(\mathrm{n}-6)$ index, that is, precursor to product of unsaturation, was used. In this way, the increase of our index with the gestational age indicates a slowing down of the $22: 4(n-6)$ desaturation process as the maturation of the children progresses.

When this index was plotted against the gestational age of the infants in TPG, a rather linear increase of the index with maturation appeared. As a first approach, then, a regression line was drawn, the correlation coefficient of which was significant at a 0.001 level. Nevertheless, as a tendency of the index to increase faster as the gestational age went up was noticed-the last cases "escaping" from the regression line on a steeper slope-a more rigorous statistical treatment was tried in order to find the curvilinear regression that better fitted the data. A quadratic regression of the type: $y=a+b x+c x^{2}$ turned out to be the best. A test of significance of departure from linear regression was performed, and the analysis of variance (based on the reduction in the sum of squares of deviations from the graph when a curved regression was used instead of a linear one) showed a value of $\mathrm{F}$ of 18.25 , significant at a $1 \%$ level. This demonstrated the much better fit of a parabolic line than that of a straight line (Fig. 1), thus proving the hypothesis that the 22:4(n-6)/22:5(n-6) index increased faster with the gestational age of the infants.

As for the mechanism of the increase of this index, it has been proposed [10-13, 15-17, 22, 27] that there is a competitive enzymatic inhibition of the desaturation between fatty acids belonging to n-3 and n- 6 series. This competitive action would be especially strong between fatty acids with the same chain length and would express the decrease of the $n-6$ fatty acid desaturation when a greater amount of $\mathbf{n}-3$ fatty acids was available as a substrate for the desaturating enzyme in the microsome (Brenner et al. [2] Galli et al. [7]). Consequently, the increase of the $n-3 / n-6$ ratio would be accompanied by an increase of the $22: 4$ (n6) $/ 22: 5(n-6)$ index, due to an inhibition of the desaturation reaction: $22: 4(n-6) \rightarrow 22: 5(n-6)$.

In a search for this cause-effect relation, the 22:4(n-6)/

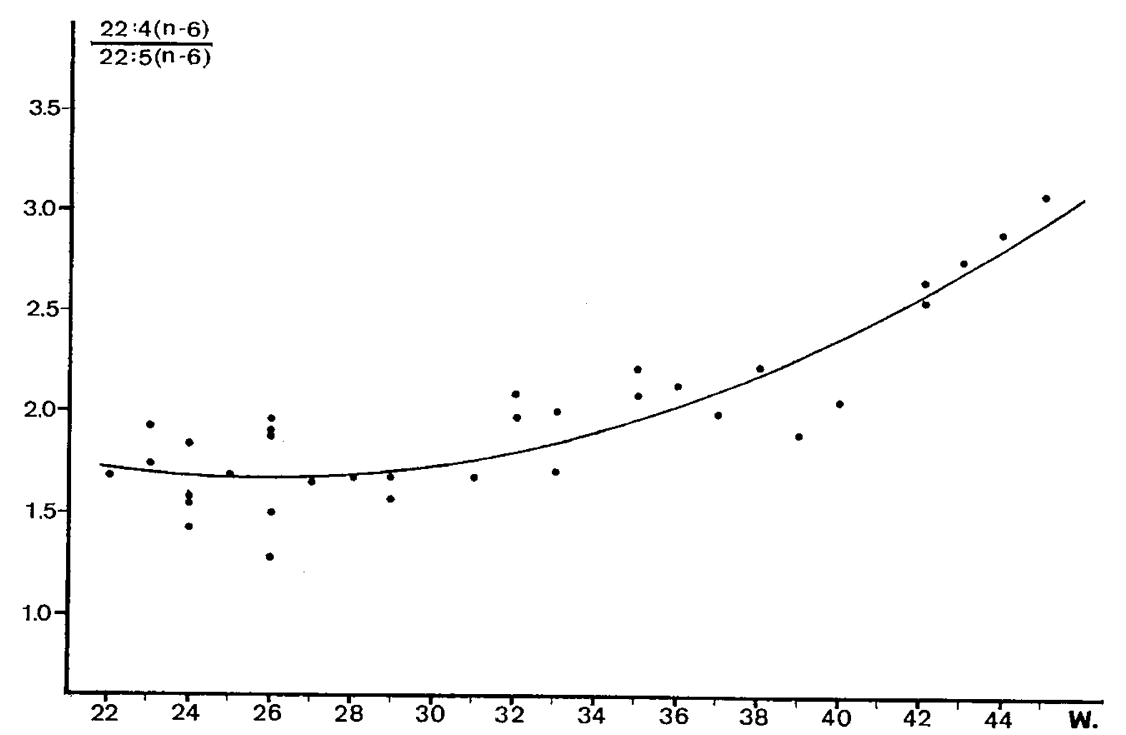

Fig. 1. Quadratic regression of the $22: 4(\mathrm{n}-6) / 22: 5(\mathrm{n}-6)$ index on gestational age in brain total phosphoglycerides: $\mathrm{y}=3.946-0.177 \mathrm{x}$ $+0.003 x^{2}$. 
22:5(n-6) index was plotted against the $n-3 / n-6$ ratio, case by case, to find out whether there was some correlation between them. Until the 40 th week of gestational age, there was no significant correlation $(\mathrm{r}=0.050, P>$ 0.1 ). Then, to look deeper into the variations of these indices, as well as to analyze other changes in the fatty acid patterns, ethanolamine and choline phosphoglycerides were studied individually.

\section{Ethanolamine Phosphoglyceride Fatty Acids}

In Tables III and IV the fatty acids of EPG in the 34 cases are shown, expressed as percentage of weight. Although on a small scale, the following changes can be seen as the maturation of the children progresses: (1) a very significant $(P<0.001)$ increase of the main n-3 series fatty acids: $22: 5(\mathrm{n}-3)$ and, especially, 22:6(n$3)$; (2) an increase of the 22:4(n-6) $(P<0.01)$, accompanied by a decrease of its product of unsaturation, 22:5(n-6), $(P<0.001) ;(3)$ an almost imperceptible, but still statistically significant $(P<0.01)$ diminution of arachidonic acid: $20: 4(\mathrm{n}-6) ;(4)$ a very slight tendency of the 18:0 to rise $(P<0.1)$, accompanied by a clearer decrease of the $\mathrm{n}-9$ fatty acids: mainly $18: 1(\mathrm{n}-9)$ $(P<0.001)$, and also $20: 3(\mathrm{n}-9)(P<0.05)$; $(5)$ a decrease of the shorter chain fatty acids: 16:0 $(P<$ $0.001)$ and $16: 1(\mathrm{n}-7)(P<0.01)$.

\section{Choline Phosphoglyceride Fatty Acids}

The fatty acid composition of CPG in all the infants can be seen in Tables $\mathrm{V}$ and VI. Within the narrow limits set by the homogeneity of the material, the following changes according to gestational age can be detected: ( 1 ) an increase of the n-3 fatty acids, much slighter than in EPG $(P<0.05)$; (2) a very small, not significant $(P>0.1)$, increase of the $22: 4(\mathrm{n}-6)$ with a remarkable decrease of the 22:5(n-6) $(P>0.001)$; (3)

Table III. Fatty acid composition of ethanolamine phosphoglycerides in newborn human brain 22-29 weeks gestational age ${ }^{1}$

\begin{tabular}{|c|c|c|c|c|c|c|c|c|c|c|c|c|c|c|c|c|c|}
\hline & $\begin{array}{c}\text { Case } 35 \\
\text { (F, } \\
22 \mathrm{wk}, \\
920 \mathrm{~g})\end{array}$ & $\begin{array}{c}\text { Case 6 } \\
\text { (M, } \\
23 \mathrm{wk}, \\
590 \mathrm{~g})\end{array}$ & $\begin{array}{c}\text { Case, } 32 \\
(\mathrm{~F}, \\
23 \mathrm{wk}, \\
1,240 \mathrm{~g})\end{array}$ & $\begin{array}{c}\text { Case } 31 \\
(\mathrm{~F}, \\
24 \mathrm{wk} \\
700 \mathrm{~g})\end{array}$ & $\begin{array}{l}\text { Case } 36 \\
(\mathrm{~F}, \\
24 \mathrm{wk} \\
800 \mathrm{~g})\end{array}$ & $\begin{array}{c}\text { Case } 38 \\
(\mathrm{~F}, \\
24 \mathrm{wk} \\
1,060 \mathrm{~g})\end{array}$ & $\begin{array}{c}\text { Case } 27 \\
(\mathrm{M}, \\
24 \mathrm{wk} \\
1,100 \mathrm{~g})\end{array}$ & $\begin{array}{c}\text { Case } 24 \\
(\mathrm{M}, \\
25 \mathrm{wk} \\
850 \mathrm{~g})\end{array}$ & $\begin{array}{c}\text { Case } 11 \\
(\mathrm{M}, \\
26 \mathrm{wk} \\
620 \mathrm{~g})\end{array}$ & $\begin{array}{c}\text { Case } 42 \\
(\mathrm{M}, \\
26 \mathrm{wk} \\
790 \mathrm{~g})\end{array}$ & $\begin{array}{c}\text { Case } 14 \\
(\mathrm{M}, \\
26 \mathrm{wk} \\
850 \mathrm{~g})\end{array}$ & $\begin{array}{c}\text { Case } 29 \\
(\mathrm{M}, \\
26 \mathrm{wk}, \\
900 \mathrm{~g})\end{array}$ & $\begin{array}{c}\text { Case } 18 \\
(\mathrm{~F}, \\
26 \mathrm{wk}, \\
1,000 \mathrm{~g})\end{array}$ & $\begin{array}{c}\text { Case } 25 \\
(\mathrm{~F}, \\
27 \mathrm{wk} \\
1,350 \mathrm{~g})\end{array}$ & $\begin{array}{c}\text { Case } 20 \\
(\mathrm{~F}, \\
28 \mathrm{wk}, \\
1,000 \mathrm{~g})\end{array}$ & $\begin{array}{c}\text { Case } 40 \\
(\mathrm{M}, \\
29 \mathrm{wk} \\
850 \mathrm{~g})\end{array}$ & $\begin{array}{c}\text { Case } 23 \\
\text { (F, } \\
29 \mathrm{wk}, \\
1,100 \mathrm{~g})\end{array}$ \\
\hline $16: 0$ & 10.32 & 11.46 & 9.03 & 11.82 & 11.10 & 9.06 & 11.64 & 11.17 & 10.90 & 10.20 & 10.13 & 9.74 & 10.14 & 11.20 & 9.22 & 9.90 & 10.73 \\
\hline $16: 1(n-7)$ & 1.13 & 1.36 & 0.87 & 1.35 & 1.20 & 1.11 & 1.32 & 1.10 & 1.14 & 1.84 & 0.82 & 1.20 & 1.00 & 1.03 & 0.74 & 0.97 & 1.12 \\
\hline $18: 0$ & 31.22 & 27.74 & 33.64 & 27.91 & 29.20 & 29.80 & 29.89 & 30.32 & 30.54 & 28.63 & 33.64 & 31.00 & 28.96 & 29.96 & 35.07 & 31.17 & 32.44 \\
\hline $18: 1(n-9)$ & 11.73 & 11.74 & 11.30 & 13.00 & 12.04 & 11.28 & 11.50 & 12.33 & 13.47 & 11.15 & 11.91 & 12.14 & 11.53 & 11.50 & 10.77 & 11.35 & 11.73 \\
\hline $18: 2(n-6)$ & 0.48 & 0.37 & 0.28 & 0.35 & 0.43 & 0.30 & 0.28 & 0.27 & 0.29 & 0.88 & 0.30 & 0.24 & 0.37 & 0.21 & 0.23 & 0.23 & 0.30 \\
\hline $\begin{array}{c}18: 3(n-3)+ \\
20: 1(n-9)\end{array}$ & 0.72 & 1.03 & 0.56 & 0.60 & 0.66 & 0.48 & 0.70 & 0.49 & 0.73 & 1.16 & 0.68 & 0.53 & 0.74 & 0.59 & 0.47 & 0.42 & 0.87 \\
\hline $20: 3(n-9)$ & 1.09 & 1.31 & 0.90 & 1.38 & 0.90 & 1.35 & 1.38 & 0.84 & 1.10 & 0.85 & 1.16 & 1.64 & 0.92 & 0.97 & 0.84 & 0.79 & 1.05 \\
\hline $20: 3(n-6)$ & 0.87 & 0.68 & 0.43 & 0.62 & 0.80 & 0.77 & 0.55 & 0.60 & 0.67 & 0.50 & 0.72 & 0.69 & 0.61 & 0.53 & 0.53 & 0.53 & 0.70 \\
\hline $20: 4(n-6)$ & 16.08 & 15.96 & 15.90 & 17.76 & 17.85 & 18.37 & 14.95 & 17.30 & 17.64 & 17.96 & 17.15 & 16.30 & 18.41 & 16.96 & 15.73 & 17.07 & 14.85 \\
\hline $22: 4(n-6)$ & 9.58 & 9.50 & 9.53 & 8.33 & 9.48 & 10.21 & 8.97 & 9.20 & 8.48 & 10.15 & 7.75 & 8.92 & 10.50 & 9.32 & 9.67 & 10.06 & 9.16 \\
\hline $22: 5(n-6)$ & 5.08 & 5.00 & 4.52 & 5.48 & 6.06 & 5.74 & 5.65 & 5.03 & 5.02 & 5.16 & 4.82 & 6.32 & 5.82 & 5.74 & 4.85 & 6.50 & 4.14 \\
\hline $22: 5(\mathrm{n}-3)$ & 0.20 & 0.40 & 0.57 & 0.30 & 0.25 & 0.43 & 0.42 & 0.37 & 0.26 & 0.37 & 0.18 & 0.33 & 0.38 & 0.39 & 0.33 & 0.22 & 0.65 \\
\hline $22: 6(n-3)$ & 11.50 & 13.45 & 12.47 & 11.10 & 10.03 & 11.10 & 12.75 & 10.98 & 9.76 & 11.15 & 10.74 & 10.95 & 10.62 & 11.60 & 11.55 & 10.79 & 12.26 \\
\hline
\end{tabular}

1 F : female; M: male.

Table IV. Fatty acid composition of ethanolamine phosphoglycerides in newborn human brain (31-45 weeks gestational age) ${ }^{1}$

\begin{tabular}{|c|c|c|c|c|c|c|c|c|c|c|c|c|c|c|c|c|c|}
\hline & $\begin{array}{c}\text { Case } 22 \\
(\mathrm{~F}, \\
31 \mathrm{wk}, \\
1,300 \mathrm{~g})\end{array}$ & $\begin{array}{c}\text { Case } 41 \\
(\mathrm{~F}, \\
32 \mathrm{wk}, \\
1,560 \mathrm{~g})\end{array}$ & $\begin{array}{c}\text { Case } 19 \\
(\mathrm{~F}, \\
32 \mathrm{wk}, \\
2,000 \mathrm{~g})\end{array}$ & $\begin{array}{c}\text { Case } 4 \\
(\mathrm{~F} \\
33 \mathrm{wk} \\
1,700 \mathrm{~g})\end{array}$ & $\begin{array}{c}\text { Case } 28 \\
(\mathrm{~F}, \\
33 \mathrm{wk}, \\
3,030 \mathrm{~g})\end{array}$ & $\begin{array}{c}\text { Case } 13 \\
(\mathrm{~F}, \\
35 \mathrm{wk}, \\
2,300 \mathrm{~g})\end{array}$ & $\begin{array}{c}\text { Case } 3 \\
(\mathrm{M}, \\
35 \mathrm{wk} \\
3,500 \mathrm{~g})\end{array}$ & $\begin{array}{c}\text { Case } 34 \\
\quad(\mathrm{~F}, \\
36 \mathrm{wk} \\
2,300 \mathrm{~g})\end{array}$ & $\begin{array}{c}\text { Case } 9 \\
(\mathrm{M}, \\
37 \mathrm{wk}, \\
2,550 \mathrm{~g})\end{array}$ & $\begin{array}{c}\text { Case 16 } \\
(\mathrm{M}, \\
38 \mathrm{wk} \\
2,850 \mathrm{~g})\end{array}$ & $\begin{array}{c}\text { Case } 7 \\
\text { (M, } \\
39 \mathrm{wk}, \\
2,970 \mathrm{~g})\end{array}$ & $\begin{array}{c}\text { Case 10 } \\
\text { (M, } \\
40 \mathrm{wk}, \\
3,400 \mathrm{~g})\end{array}$ & $\begin{array}{c}\text { Case } 30 \\
(\mathrm{M}, \\
42 \mathrm{wk}, \\
3,025 \mathrm{~g})\end{array}$ & $\begin{array}{c}\text { Case } 33 \\
(\mathrm{~F}, \\
42 \mathrm{wk} \\
4,140 \mathrm{~g})\end{array}$ & $\begin{array}{c}\text { Case } 8 \\
(\mathrm{M}, \\
43 \mathrm{wk}, \\
3,500 \mathrm{~g})\end{array}$ & $\begin{array}{c}\text { Case } 5 \\
\text { (F, } \\
44 \mathrm{wk}, \\
4,100 \mathrm{~g})\end{array}$ & $\begin{array}{c}\text { Case 21 } \\
\text { (F, } \\
45 \mathrm{wk}, \\
3,500 \mathrm{~g})\end{array}$ \\
\hline $16: 0$ & 9.03 & 10.82 & 8.40 & 10.74 & 8.87 & 11.62 & 7.61 & 8.30 & 8.47 & 9.34 & 10.53 & 7.70 & 10.62 & 7.71 & 7.37 & 8.74 & 9.20 \\
\hline $16: 1(n-7)$ & 0.86 & 1.50 & 0.63 & 1.11 & 1.00 & 0.92 & 0.91 & 0.93 & 0.89 & 1.33 & 0.95 & 0.58 & 1.08 & 1.02 & 0.51 & 0.74 & 1.06 \\
\hline $18: 0$ & 32.59 & 29.43 & 34.08 & 28.30 & 29.87 & 32.86 & 31.44 & 33.58 & 32.36 & 33.38 & 30.00 & 29.72 & 29.63 & 34.47 & 32.55 & 31.74 & 31.90 \\
\hline $18: 1(n-9)$ & 10.96 & 10.00 & 7.92 & 10.30 & 8.54 & 10.11 & 8.91 & 10.78 & 8.88 & 10.30 & 10.29 & 7.74 & 9.30 & 10.37 & 8.74 & 9.10 & 10.84 \\
\hline $18: 2(n-6)$ & 0.21 & 0.40 & 0.16 & 0.27 & 0.26 & 0.16 & 0.26 & 0.20 & 0.23 & 0.71 & 0.36 & 0.16 & 0.26 & 0.18 & 0.16 & 0.19 & 0.20 \\
\hline $\begin{array}{c}18: 3(n-3)+ \\
20: 1(n-9)\end{array}$ & 0.54 & 0.45 & 0.36 & 0.57 & 0.69 & 0.45 & 0.46 & 0.42 & 0.45 & 0.78 & 0.68 & 0.46 & 0.64 & 0.60 & 0.39 & 0.66 & 0.65 \\
\hline $20: 3(n-9)$ & 0.79 & 1.75 & 0.93 & 1.18 & 1.66 & 0.70 & 0.81 & 0.90 & 1.00 & 0.87 & 0.87 & 1.10 & 0.60 & 0.94 & 0.70 & 1.06 & 0.91 \\
\hline $20: 3(n-6)$ & 0.66 & 0.72 & 0.80 & 0.82 & 0.84 & 0.89 & 0.96 & 0.79 & 0.82 & 0.93 & 0.83 & 0.90 & 1.00 & 0.86 & 1.00 & 1.10 & 1.20 \\
\hline $20: 4(n-6)$ & 17.42 & 15.20 & 14.90 & 17.65 & 17.73 & 15.25 & 16.20 & 16.26 & 16.70 & 14.37 & 15.53 & 16.16 & 15.83 & 14.82 & 15.90 & 15.25 & 14.47 \\
\hline $22: 4(n-6)$ & 10.42 & 10.08 & 11.97 & 10.50 & 11.24 & 10.00 & 12.04 & 11.35 & 11.80 & 11.17 & 10.61 & 11.83 & 10.93 & 10.36 & 12.58 & 11.38 & 10.72 \\
\hline $22: 5(n-6)$ & 5.32 & 6.00 & 5.43 & 4.74 & 6.27 & 4.62 & 4.20 & 4.52 & 5.27 & 4.89 & 5.27 & 5.95 & 4.13 & 3.55 & 3.97 & 3.10 & 2.62 \\
\hline $22: 5(n-3)$ & 0.35 & 0.45 & 0.22 & 0.21 & 0.20 & 0.30 & 0.45 & 0.18 & 0.33 & 0.87 & 0.46 & 0.40 & 0.48 & 0.50 & 0.48 & 0.68 & 0.75 \\
\hline $22: 6(n-3)$ & 10.85 & 13.20 & 14.20 & 13.61 & 12.83 & 12.12 & 15.75 & 11.79 & 12.80 & 11.06 & 13.62 & 17.30 & 15.50 & 14.62 & 15.65 & 16.26 & 15.48 \\
\hline
\end{tabular}

$1 \mathrm{~F}$ : female; $\mathrm{M}$ : male. 
Table $V$. Fatty acid composition of choline phosphoglycerides in newborn human brain (22-29 weeks gestational age $)^{1}$

\begin{tabular}{|c|c|c|c|c|c|c|c|c|c|c|c|c|c|c|c|c|c|}
\hline & $\begin{array}{c}\text { Case } 35 \\
(\mathrm{~F}, \\
22 \mathrm{wk} \\
920 \mathrm{~g})\end{array}$ & $\begin{array}{c}\text { Case } 6 \\
(\mathrm{M}, \\
23 \mathrm{wk} \\
590 \mathrm{~g})\end{array}$ & $\begin{array}{c}\text { Case } 32 \\
(\mathrm{~F}, \\
23 \mathrm{wk} \\
1,240 \mathrm{~g})\end{array}$ & $\begin{array}{c}\text { Case } 31 \\
(\mathrm{~F}, \\
24 \mathrm{wk}, \\
700 \mathrm{~g})\end{array}$ & $\begin{array}{c}1 \text { Case } 36 \\
(\mathrm{~F}, \\
24 \mathrm{wk} \\
800 \mathrm{~g})\end{array}$ & $\begin{array}{c}\text { Case } 38 \\
(\mathrm{~F}, \\
24 \mathrm{wk} \\
1,060 \mathrm{~g})\end{array}$ & $\begin{array}{c}\text { Case } 27 \\
(\mathrm{M}, \\
24 \mathrm{wk}, \\
1,100 \mathrm{~g})\end{array}$ & $\begin{array}{c}\text { Case } 24 \\
(\mathrm{M}, \\
25 \mathrm{wk}, \\
850 \mathrm{~g})\end{array}$ & $\begin{array}{c}4 \text { Case } 11 \\
(\mathrm{M}, \\
26 \mathrm{wk}, \\
620 \mathrm{~g})\end{array}$ & $\begin{array}{c}1 \text { Case } 42 \\
(\mathrm{M}, \\
26 \mathrm{wk} \\
790 \mathrm{~g})\end{array}$ & $\begin{array}{c}\text { Case } 14 \\
(\mathrm{M}, \\
26 \mathrm{wk}, \\
850 \mathrm{~g})\end{array}$ & $\begin{array}{c}\text { Case } 29 \\
(\mathrm{M}, \\
26 \mathrm{wk}, \\
900 \mathrm{~g})\end{array}$ & $\begin{array}{c}\text { Case 18 } \\
(\mathrm{F}, \\
26 \mathrm{wk} \\
1,000 \mathrm{~g})\end{array}$ & $\begin{array}{c}\text { Case } 25 \\
(\mathrm{~F}, \\
27 \mathrm{wk} \\
1,350 \mathrm{~g})\end{array}$ & $\begin{array}{c}\text { Case } 20 \\
(\mathrm{~F}, \\
28 \mathrm{wk}, \\
1,000 \mathrm{~g})\end{array}$ & $\begin{array}{c}\text { Case } 40 \\
(\mathrm{M}, \\
29 \mathrm{wk} \\
850 \mathrm{~g})\end{array}$ & $\begin{array}{c}\text { Case } 23 \\
(\mathrm{~F}, \\
29 \mathrm{wk}, \\
1,100 \mathrm{~g})\end{array}$ \\
\hline $14: 0$ & 3.55 & 4.27 & 3.90 & 4.12 & 4.18 & 4.06 & 3.91 & 4.47 & 4.50 & 4.57 & 3.76 & 3.97 & 4.70 & 4.19 & 3.73 & 4.06 & 3.33 \\
\hline $16: 0$ & 51.38 & 51.48 & 51.52 & 50.25 & 50.98 & 50.95 & 51.77 & 51.90 & 50.55 & 53.24 & 51.22 & 50.62 & 51.38 & 51.76 & 52.57 & 52.08 & 51.29 \\
\hline $16: 1(n-7)$ & 6.36 & 6.64 & 6.10 & 7.25 & 6.80 & 7.04 & 6.77 & 7.27 & 7.27 & 7.33 & 6.31 & 7.06 & 6.30 & 6.91 & 6.90 & 6.40 & 6.23 \\
\hline $18: 0$ & 7.95 & 6.60 & 7.70 & 7.16 & 7.14 & 7.56 & 7.61 & 7.05 & 7.25 & 6.49 & 7.58 & 7.16 & 6.96 & 7.22 & 7.53 & 7.60 & 7.73 \\
\hline $18: 1(n-9)$ & 22.43 & 22.46 & 22.05 & 22.96 & 22.62 & 22.42 & 21.85 & 22.10 & 23.15 & 21.30 & 22.66 & 23.27 & 22.08 & 21.53 & 21.73 & 21.53 & 23.36 \\
\hline $18: 2(n-6)$ & 0.50 & 0.52 & 0.72 & 0.47 & 0.70 & 0.52 & 0.46 & 0.41 & 0.55 & 0.50 & 0.39 & 0.47 & 0.50 & 0.45 & 0.29 & 0.54 & 0.48 \\
\hline $\begin{array}{c}18: 3(\mathrm{n}-3)+ \\
20: 1(\mathrm{n}-9)\end{array}$ & 0.80 & 0.69 & 0.77 & 0.61 & 0.55 & 0.50 & 0.69 & 0.50 & 0.41 & 0.64 & 0.63 & 0.61 & 0.54 & 0.63 & 0.55 & 0.46 & 0.54 \\
\hline $20: 3(n-9)$ & 0.13 & 0.29 & 0.18 & 0.25 & 0.13 & 0.21 & 0.25 & 0.17 & 0.17 & 0.18 & 0.30 & 0.31 & 0.14 & 0.14 & 0.15 & 0.19 & 0.21 \\
\hline $20: 3(n-6)$ & 0.38 & 0.32 & 0.33 & 0.31 & 0.43 & 0.34 & 0.24 & 0.31 & 0.32 & 0.32 & 0.45 & 0.32 & 0.30 & 0.28 & 0.28 & 0.29 & 0.30 \\
\hline $20: 4(n-6)$ & 4.15 & 3.94 & 4.08 & 4.17 & 4.50 & 4.41 & 4.21 & 3.80 & 3.95 & 3.88 & 4.27 & 3.88 & 4.32 & 4.18 & 4.17 & 4.23 & 4.03 \\
\hline $22: 4(n-6)$ & 0.55 & 0.90 & 0.83 & 0.77 & 0.75 & 0.61 & 0.73 & 0.71 & 0.67 & 0.59 & 0.67 & 0.73 & 1.20 & 0.80 & 0.63 & 0.88 & 0.75 \\
\hline $22: 5(n-6)$ & 0.40 & 0.37 & 0.62 & 0.47 & 0.37 & 0.33 & 0.41 & 0.32 & 0.37 & 0.32 & 0.41 & 0.64 & 0.53 & 0.44 & 0.29 & 0.60 & 0.38 \\
\hline $22: 5(n-3)$ & 0.17 & 0.10 & tr. & 0.07 & tr. & tr. & tr. & 0.07 & tr. & tr. & 0.18 & tr. & tr. & 0.11 & tr. & 0.06 & 0.13 \\
\hline $22: 6(n-3)$ & 1.25 & 1.42 & 1.20 & 1.14 & 0.85 & 1.05 & 1.10 & 0.92 & 0.84 & 0.64 & 1.17 & 0.96 & 1.05 & 1.36 & 1.18 & 1.08 & 1.24 \\
\hline
\end{tabular}

Table VI. Fatty acid composition of choline phosphoglycerides in newborn human brain (31-45 weeks gestational age $)^{1}$

\begin{tabular}{|c|c|c|c|c|c|c|c|c|c|c|c|c|c|c|c|c|c|}
\hline & $\begin{array}{c}\text { Case } 22 \\
(\mathrm{~F}, \\
31 \mathrm{wk}, \\
1,300 \mathrm{~g})\end{array}$ & $\begin{array}{c}\text { Case 41 } \\
(\mathrm{F}, \\
32 \mathrm{wk} \\
1,560 \mathrm{~g})\end{array}$ & $\begin{array}{c}\text { Case } 19 \\
(\mathrm{~F}, \\
32 \mathrm{wk} \\
2,000 \mathrm{~g})\end{array}$ & $\begin{array}{c}\text { Case } 4 \\
(\mathrm{~F}, \\
33 \mathrm{wk}, \\
1,700 \mathrm{~g})\end{array}$ & $\begin{array}{c}\text { Case } 28 \\
(\mathrm{~F}, \\
33 \mathrm{wk}, \\
3,030 \mathrm{~g})\end{array}$ & $\begin{array}{c}\text { Case } 13 \\
(\mathrm{~F}, \\
35 \mathrm{wk} \\
2,300 \mathrm{~g})\end{array}$ & $\begin{array}{c}\text { Case } 3 \\
\text { (M, } \\
35 \mathrm{wk} \\
3,500 \mathrm{~g})\end{array}$ & $\begin{array}{c}\text { Case } 34 \\
(\mathrm{~F}, \\
36 \mathrm{wk} \\
2,300 \mathrm{~g})\end{array}$ & $\begin{array}{c}\text { Case } 9 \\
(\mathrm{M}, \\
37 \mathrm{wk} \\
2,550 \mathrm{~g})\end{array}$ & $\begin{array}{c}\text { Case 16 } \\
(\mathrm{M}, \\
38 \mathrm{wk} \\
2,850 \mathrm{~g})\end{array}$ & $\begin{array}{c}\text { Case } 7 \\
(\mathrm{M}, \\
39 \mathrm{wk} \\
2,970 \mathrm{~g})\end{array}$ & $\begin{array}{l}\text { Case } 10 \\
\text { (M, } \\
40 \mathrm{wk}, \\
3,400 \mathrm{~g})\end{array}$ & $\begin{array}{c}\text { Case } 30 \\
\text { (M, } \\
42 \mathrm{wk}, \\
3,025 \mathrm{~g})\end{array}$ & $\begin{array}{c}\text { Case } 33 \\
(\mathrm{~F}, \\
42 \mathrm{wk} \\
4,140 \mathrm{~g})\end{array}$ & $\begin{array}{c}\text { Case } 8 \\
(\mathrm{M}, \\
43 \mathrm{wk} \\
3,500 \mathrm{~g}(\end{array}$ & $\begin{array}{c}\text { Case } 5 \\
(\mathrm{~F}, \\
44 \mathrm{wk} \\
4,100 \mathrm{~g})\end{array}$ & $\begin{array}{l}\text { Case } 21 \\
(\mathrm{~F}, \\
45 \mathrm{wk}, \\
3,500 \mathrm{~g})\end{array}$ \\
\hline $14: 0$ & 3.67 & 3.72 & 2.44 & 3.68 & 3.60 & 3.22 & 2.54 & 3.00 & 2.77 & 3.53 & 3.08 & 2.74 & 3.60 & 2.68 & 2.11 & 1.87 & 2.48 \\
\hline $16: 0$ & 52.84 & 47.86 & 52.38 & 50.88 & 50.37 & 54.72 & 49.30 & 52.20 & 51.78 & 53.05 & 52.22 & 51.24 & 49.98 & 49.68 & 51.54 & 50.76 & 52.03 \\
\hline $16: 1(n-7)$ & 6.41 & 7.90 & 5.61 & 6.61 & 6.76 & 5.27 & 6.58 & 6.58 & 5.70 & 6.56 & 6.43 & 5.87 & 6.38 & 6.43 & 5.20 & 4.96 & 6.43 \\
\hline $18: 0$ & 7.36 & 8.53 & 8.98 & 7.43 & 8.00 & 8.53 & 8.45 & 7.76 & 8.16 & 7.71 & 7.43 & 8.32 & 8.44 & 8.77 & 9.10 & 9.89 & 8.00 \\
\hline $18: 1(n-9)$ & 22.00 & 22.10 & 21.78 & 22.57 & 20.83 & 21.53 & 23.35 & 22.52 & 22.64 & 21.15 & 21.61 & 22.06 & 21.60 & 23.82 & 22.65 & 23.11 & 22.46 \\
\hline $18: 2(n-6)$ & 0.38 & 0.64 & 0.48 & 0.48 & 0.57 & 0.37 & 0.54 & 0.50 & 0.49 & 0.46 & 0.38 & 0.47 & 0.40 & 0.44 & 0.27 & 0.35 & 0.58 \\
\hline $\begin{array}{c}18: 3(\mathrm{n}-3)+ \\
20: 1(\mathrm{n}-9)\end{array}$ & 0.51 & 0.53 & 0.43 & 0.61 & 0.58 & 0.45 & 0.62 & 0.52 & 0.53 & 0.53 & 0.60 & 0.70 & 0.56 & 0.51 & 0.38 & 0.46 & 0.51 \\
\hline $20: 3(n-9)$ & 0.14 & 0.33 & 0.17 & 0.25 & 0.34 & 0.10 & 0.23 & 0.20 & 0.16 & 0.11 & 0.22 & 0.28 & 0.13 & 0.20 & 0.18 & 0.21 & 0.18 \\
\hline $20: 3(n-6)$ & 0.33 & 0.33 & 0.43 & 0.43 & 0.51 & 0.35 & 0.60 & 0.40 & 0.43 & 0.43 & 0.47 & 0.50 & 0.55 & 0.47 & 0.58 & 0.67 & 0.64 \\
\hline $20: 4(n-6)$ & 4.25 & 4.88 & 4.81 & 4.68 & 5.86 & 3.74 & 5.24 & 4.43 & 5.13 & 4.43 & 4.90 & 5.20 & 5.58 & 4.57 & 5.37 & 5.12 & 4.59 \\
\hline $22: 4(n-6)$ & 0.72 & 1.00 & 0.86 & 0.82 & 0.88 & 0.64 & 0.78 & 0.66 & 0.75 & 0.76 & 1.00 & 0.77 & 0.92 & 0.69 & 0.88 & 1.10 & 0.59 \\
\hline $22: 5(n-6)$ & 0.30 & 0.57 & 0.35 & 0.32 & 0.50 & 0.26 & 0.26 & 0.26 & 0.29 & 0.33 & 0.34 & 0.40 & 0.29 & 0.31 & 0.19 & 0.13 & 0.18 \\
\hline $22: 5(n-3)$ & 0.11 & 0.08 & tr. & 0.24 & tr. & tr. & 0.07 & tr. & 0.13 & 0.14 & tr. & 0.20 & 0.13 & 0.11 & 0.14 & 0.07 & 0.08 \\
\hline $22: 6(n-6)$ & 0.98 & 1.53 & 1.28 & 1.00 & 1.20 & 0.82 & 1.44 & 0.97 & 1.04 & 0.81 & 1.32 & 1.25 & 1.44 & 1.32 & 1.41 & 1.30 & 1.25 \\
\hline
\end{tabular}

the sum of the fatty acids of the linoleic family rises clearly, mainly due to the increase of the 20:4(n-6) which is statistically very significant $(P<0.001)$; (4) an increase of stearic acid (18:0), very significant $(P<$ $0.001)$, but not accompanied in this case by a decrease of any of the n-9 fatty acids, which do not show appreciable changes during this period of life in CPG; (5) the shortest chain fatty acid, myristic acid (14:0), shows a clear diminution $(P<0.001)$; as does the $16: 1(n-7)$. Palmitic acid (16:0), on the other hand, does not change during this period.

As the relation between the different fatty acids is important, a series of indices were again studied in EPG and CPG in order to better evaluate the changes in the fatty acid patterns in each individual case.

\section{n-3/n-6 Index}

When this index was plotted against the gestational age in EPG a clear increase of the $n-3 / n-6$ ratio appeared, almost exclusively due to a rise in 22:6(n-3). The increase was parabolic rather than linear (Fig. 2), the closeness of fit of the quadratic regression being at a $1 \%$ significance level when tested against a linear regression $(F=10.58)$.

In the graph of the same index for $C P G$, however, there was no significant variation of the $n-3 / n-6$ ratio during the span of gestational ages, as shown by a correlation coefficient of only 0.060 . This was due to the increase of the 20:4(n-6) in choline phosphoglycerides which compensated for the small increase of the numerator 22:6(n-3) and 22:5(n-3). 


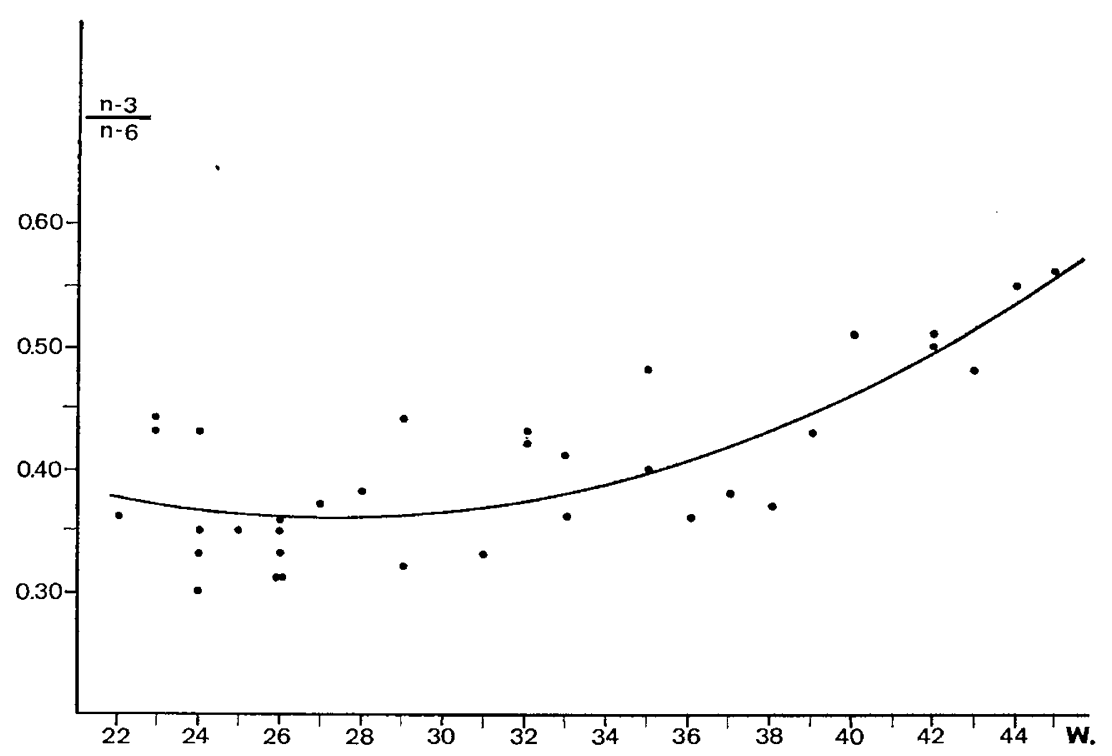

Fig. 2. Fitted curve of the $\mathrm{n}-3 / \mathrm{n}-6$ ratio against gestational age in brain ethanolamine phosphoglycerides. The parabola is: $y=0.807-$ $0.033 \mathrm{x}+0.0006 \mathrm{x}^{2}$.

\section{$22: 4(n-6) / 22: 5(n-6)$ Index}

In EPG the increase of this index when plotted against the gestational age was remarkable. To this increase both numerator and denominator contributed very significantly, the former by rising and the latter by falling. In the graph the same phenomenon already observed in TPG, namely, the sharper slope of the curve in the older children could be seen even more clearly. A curvilinear regression was tried and a quadratic one was found to fit the best (Fig. 3), the curvilinearity of the regression being significant at a $1 \%$ level ( $F=16.43$ in the test of significance of departure from linear regression).

As shown in the three parabolic graphs, the ratios do not increase at all until the 29th-31st week. This is in agreement with the findings previously reported [3], and would again suggest that the brain maturation

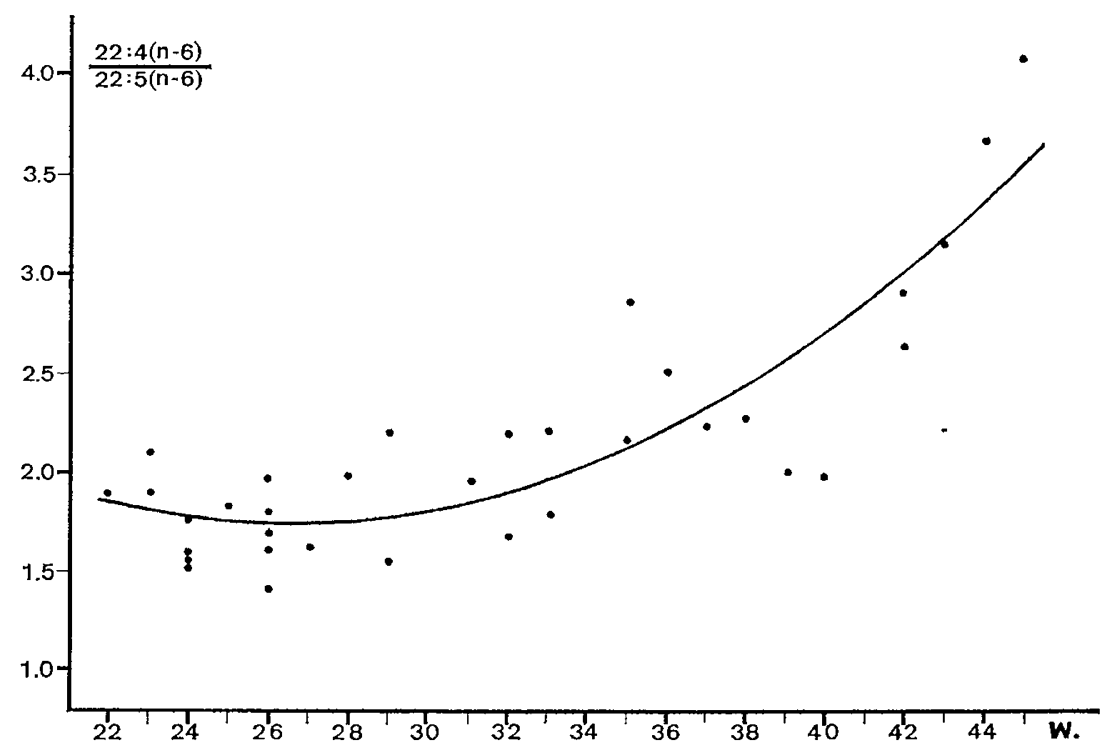

Fig. 3. Parabolic regression of the $22: 4(n-6) / 22: 5(n-6)$ index on gestational age in brain ethanolamine phosphoglycerides: $y=5.560-$ $0.286 \mathrm{x}+0.005 \mathrm{x}^{2}$. 
begins to be important after the 31 st week of intrauterine life, when the fetus is already viable. Then the increase of these ratios becomes faster and faster as the gestation approaches its term.

When the 22:4(n-6)/22:5(n-6) ratio was plotted against gestational age in CPG a much slower but still significant increase $(P<0.001)$ was found. There was also here a tendency of the index to increase faster towards the end of gestation.

For the purpose of studying deeper the competitive enzymatic inhibition between the $n-3$ and $n-6$ families of fatty acids, the $22: 4(\mathrm{n}-6) / 22: 5(\mathrm{n}-6)$ index was plotted against the $n-3 / n-6$ ratio, case by case, in EPG and CPG. In ethanolamine phosphoglycerides, in spite of the dispersion of the values, a very significant quadratic regression $(\mathrm{F}=11.00,1 \%$ level of significance) was found between both indices. This suggests that the n-3 fatty acids need to reach a certain level to inhibit the desaturation of the 22:4(n-6) and then the enzymatic inhibition progresses in a quadratic manner. It would explain the stronger increase of the $22: 4(\mathrm{n}-6) / 22: 5(\mathrm{n}$ 6 ) index towards the end of gestation, when the ratio between the $n-3$ and $n-6$ families of fatty acids has already reached a good level and is increasing faster.

$[22: 4(n-6)+22: 5(n-6)] / 20: 4(n-6)$ Index

When the elongation process of arachidonic acid was analyzed, it emerged that the $22: 4(n-6) / 20: 4(n-6)$ ratio, reported as representative of the $20: 4(n-6)$ elongation $[7,20]$ would have given us an idea not just of the chain-lengthening process of arachidonic acid. It is, in fact, the exponent of two different processes at the same time: the elongation of the 20:4(n-6) to $22: 4(n-6)$ and the inhibition of the desaturation of the $22: 4(\mathrm{n}-6)$ to $22: 5(\mathrm{n}-6)$. In other words, the increase of the $22: 4(\mathrm{n}-6)$ can be caused not only by an elongation of the $20: 4(\mathrm{n}-6)$ to $22: 4(\mathrm{n}-6)$ but also, and even in a greater proportion in our cases, by a slowing down of the 22:4(n-6) desaturation to $22: 5(\mathrm{n}-6)$. That was evident in this report because the increase of the $22: 4(\mathrm{n}$ 6) was always accompanied by a decrease of its product of unsaturation, 22:5(n-6), and the sum of both only underwent a very slight increase in EPG $(r=0.245)$ and a small decrease in CPG $(\mathrm{r}=-0.188)$, both without statistical significance. Therefore, this sum would be a better measurement of the chain-lengthening reaction of the 20:4(n-6) because not only the 22:4(n-6) but also the 22:5(n-6) are products of arachidonic acid, and the sum of both has the advantage of not mixing the significant changes between these two fatty acids.
Thus, in order to see what the 20:4(n-6) elongation process was like in the present group of newborn infants, the 22:4(n-6) and the 22:5(n-6) were added together and the result divided by the arachidonic acid, that is: $[22: 4(\mathrm{n}-6)+22: 5(\mathrm{n}-6)] / 20: 4(\mathrm{n}-6)$. In this way, the influence of the $22: 4(\mathrm{n}-6) \rightarrow 22: 5(\mathrm{n}-6)$ reaction was automatically eliminated and a more reliable estimate of the elongation of arachidonic acid was obtained.

With the help of this index, a rather linear increase of the 20:4(n-6) elongation was found in ethanolamine phosphoglycerides as the gestational age of the children went up $(P<0.005)$. In choline phosphoglycerides, on the other hand, the chain-lengthening of arachidonic acid decreased as the gestational age of the infants rose, and the significance level was higher $(P<$ $0.001)$.

$n-6 / n-9$ Index

The sum of the fatty acids of the linoleic family was divided by the sum of those of the oleic family to see if the ratio between the two series of fatty acids changed during this period of intrauterine life. A fairly linear increase of the $n-6 / n-9$ index was found in both CPG and EPG as gestation progressed. The statistical significance level was 0.001 in EPG and 0.01 in CPG.

18:0/18:1(n-9)

When this ratio was plotted against the gestational age of the children, a clearly linear positive correlation was found in both EPG (Fig. 4) and CPG between the maturation of the infants and the stearate to oleate ratio. The level of significance was 0.001 in both cases. Nevertheless, in ethanolamine phosphoglycerides the rise of the index was due to a decrease of oleic acid, whereas in choline phosphoglycerides this rise was caused by an increase of stearic acid. In addition, the dispersion of the values was greater in CPG than in EPG.

\section{Summary}

The fatty acids of brain phosphoglycerides were analyzed by gas chromatography in a homogeneous group of 34 newborn babies with different degree of maturation. The length of gestation ranged from 22 to 45 weeks. All the children had died within the first hours of life, before feeding, and none showed any sign of cerebral damage. After a primary orientation study of the total phosphoglycerides, the fatty acids of ethanolamine and choline phosphoglycerides were analyzed 


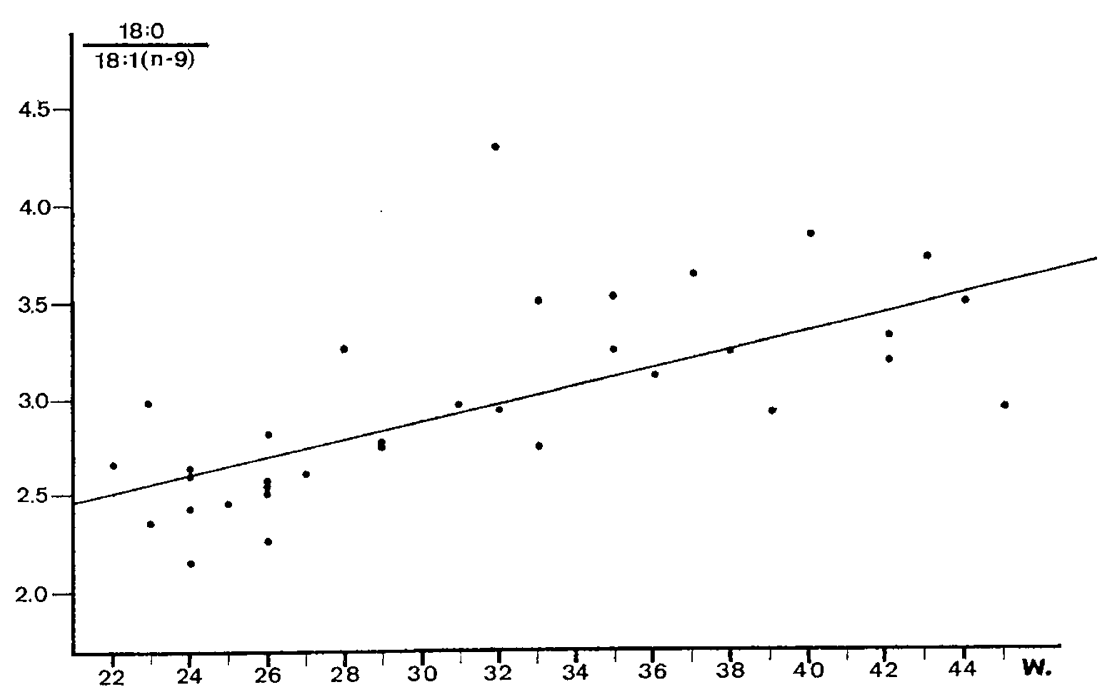

Fig. 4. Linear increase of the 18:0/18:1(n-9) index with the gestational age in brain ethanolamine phosphoglycerides. The equation of the regression line is: $\mathrm{y}=1.475+0.047 \mathrm{x}$.

more deeply. The main changes found as gestation progressed can be summarized as follows. The fatty acids of the linolenic ( $n-3)$ family increased, more in EPG than in GPG, whereas those of the oleic (n-9) family decreased in EPG and did not change in CPG. As for the fatty acids of the linoleic (n-6) series, although their sum did not vary significantly in EPG, the individual fatty acids underwent definite changes with maturation. The most important was a decrease of the 22:5(n-6) in both phosphoglycerides, accompanied by an increase of its precursor, $22: 4(\mathrm{n}-6)$, only significant in EPG. As a result, a marked increase of the $22: 4(\mathrm{n}-6) / 22: 5(\mathrm{n}-6)$ ratio was found as with longer gestation, the increase following a parabolic profile. The arachidonic acid $(20: 4(\mathrm{n}-6))$, which did not vary in TPG, showed a decrease in EPG and an increase in CPG, as a result of the activation or inhibition, respectively, of its elongation to higher members of the series as maturation progressed. This was shown by the changes of the $[22: 4(n-6)+22: 5(n-6)] / 20: 4(n-6)$ index. Among the saturated fatty acids, there was a decrease of the shorter ones, not equal in each phosphoglyceride. Myristic acid (14:0) decreased remarkably in TPG and CPG, but palmitic acid (16:0) only fell in EPG, and stearic acid (18:0) rose strongly in CPG and much less in EPG. Finally, the monoenes, palmitoleic and oleic acids (16:1(n-7) and 18:1(n-9)), decreased clearly in EPG, especially the latter, whilst in CPG only palmitoleic acid diminished. In spite of this, the 18:0/18:1(n-9) ratio rose linearly with gestational age in both phosphoglycerides because of the higher increase of stearic acid in CPG than in EPG.

\section{References and Notes}

1. Bernsohn, J., AND Cohen, S. R.: Polyenoic and fatty acid metabolism of phosphoglycerides in developing brain. Lipids, Malnutrition and the Developing Brain, p. 159 (Ciba Foundation Symposium, Elsevier, Amsterdam, 1972).

2. Brenner, R. R., de Tomas, M. E., and Peluffo, R. O.: Effect of polyunsaturated fatty acids on the desaturation in vitro of linoleic to $\gamma$-linolenic acid. Biochim. Biophys. Acta, 106: 640 (1965).

3. Conde, C., Martinez, M., And Ballabriga, A.: Some chemical aspects of human brain development. I. Neutral glycosphingolipids, sulfatides, and sphingomyelin. Pediat. Res., 8: \$\$ (1974).

4. Davson, H.: The blood brain barrier. Advan. Exp. Med. Biol., 13: 381 (1971).

5. Dhopeshwarkar, G. A., AND Mead, J. F.: Role of oleic acid in the metabolism of essential fatty acids. J. Amer. Oil Chem. Soc., 38: 297 (1961).

6. Fisher, R. A., AND YATes, F.: Statistical Tables for Biological, Agricultural and Medical Research (Oliver Boyd, Edinburgh, 1963).

7. Gally, C., Trzeciak, H. I., and Paoletti, R.: Effects of dietary fatty acids on the fatty acid composition of brain ethanolamine phosphoglyceride: Reciprocal replacement of $\mathrm{n}-6$ and $\mathrm{n}-3$ polyunsaturated fatty acids. Biochim. Biophys. Acta, 248: 449 (1971).

8. Holman, R. T.: The ratio of trienoic:tetraenoic acids in tissue lipids as a measure of essential fatty acid requirement. $\mathrm{J}$. Nutr., 70: 405 (1960).

9. Holman, R. T.: Polyunsaturated acids. Progr. Chem. Fats Other Lipids, 9: 324 (1971). 
10. KLENK, E., AND OETTE, K.: The C20- and C22-polyenoic acids of rat liver phosphatides after addition of linoleic and linolenic acid to a fat-free diet. Z. Physiol. Chem., 318: 86 (1960).

11. MAGhlin, L. J., AND Gordon, R. S.: Effect of dietary fatty acids and cholesterol on growth and fatty acid composition of the chicken. J. Nutr., 75: 157 (1961).

12. Machlin, L. J.: Effect of dietary linolenate on the proportion of linoleate and arachidonate in liver fat. Nature, 194: 868 (1962).

13. Marco, G. J., Machlin, L. J., Emery, E., and Gordon, R. S.: Dietary effects of fats upon fatty acid composition of the mitochondria. Arch. Biochem. Biophys., 94: 115 (1961).

14. Mead, J. F., AND Dhopeshwarkar, G. A.: Types of fatty acids in brain lipids, their derivation and function. Lipids, Malnutrition and the Developing Brain, p. 59 (Ciba Foundation Symposium, Elsevier, Amsterdam, 1972).

15. Mohrhauer, H., and Holman, R. T.: The effect of dose level of essential fatty acids upon fatty acid composition of the rat liver. J. Lipid Res., 4: 151 (1963).

16. Mohrhauer, H., and Holman, R. T.: Effect of linolenic acid upon the metabolism of linoleic acid. J. Nutr., 81: 67 (1963).

17. Mohrhauer, H., and Holman, R. T.: Alteration of the fatty acid composition of brain lipids by varying levels of dietary essential fatty acids. J. Neurochem., 10:523 (1963).

18. O'Brien, J. S., Fullerup, D. L., ANd Mead, J. F.: Quantification and fatty acid and fatty aldehyde composition of ethanolamine, choline, and serine glycerophosphatides in human cerebral grey and white matter. J. Lipid Res., 5: 329 (1964).

19. O'Brien, J. S., ANd SAmpson, E. L.: Fatty acid and fatty aldehyde composition of the major brain lipids in normal human gxay matter, white matter, and myelin. J. Lipid Res., 6: 545 (1965).

20. Paoletri, R., ANd Galli, C.: Effects of essential fatty acid deficiency on the central nervous system in the growing rat. Lipids, Malnutrition and the Developing Brain, p. 121 (Ciba Foundation Symposium, Elsevier, Amsterdam, 1972).

21. RaHM, J. J., and Holman, R. T.: The relationship of single dietary polyunsaturated fatty acids to fatty acid composition of lipids from subcellular particles of liver. J. Lipid Res., 5: 169 (1964).
22. Rahm, J. J., and Holman, R. T.: Effect of linoleic acid upon the metabolism of linolenic acid. J. Nutr., 84: 15 (1964).

23. SNedecor, G. W., and Cochram, W. G.: Statistical Methods (The lowa University Press, Ames, Iowa, 1967).

24. SvenNerholm, L.: Distribution and fatty acid composition of phosphoglycerides in normal human brain. J. Lipid Res., 9: 570 (1968).

25. Svennerholm, L., Alling, C., Bruce, A., Karlsson, I., AND SAPIA, O.: Effects on offspring of maternal malnutrition in the rat. Lipids, Malnutrition and the Developing Brain, p. I4I (Ciba Foundation Symposium, Elsevier, Amsterdam, 1972).

26. Svennerholm, L., AND VANier, O.: The distribution of lipids in the human nervous system. III. Fatty acid composition of phosphoglycerides of human foetal and infant brain. Brain Res., 50: 341 (1973).

27. White, H. B., Galli, C., ANd Paoletti, R.: Brain recovery from essential fatty acid deficiency in developing rats. J. Neurochem., 18: 869 (1971).

28. White, H. B., Galli, C., and Paoletti, R.: Ethanolamine phosphoglyceride fatty acids in aging human brain. J. Neurochem., 18: 1337 (1971).

29. The symbol $\mathrm{n}$ and the figure that follows refer to the position of the first double bond from the methyl group. The others are methylene-interrupted double bonds added in the direction of the carboxyl group according to the number placed after that of carbon atoms: $20: 4(n-6)$, for example, will mean a fatty acid with 20 carbon atoms and 4 double bonds in positions $6,9,12$, and 15 , counting the methyl group as number one.

30. While the present paper was in press, a study by Svennerholm and Vanier [26] on brains of 11 fetuses, 8 neonates, and 13 infants until 2 years of age appeared. The authors found an increase of the 22:6(n-3) and 18:0 as well as of the elongation and desaturation of the $n-6$ fatty acids during the prenatal life.

31. Requests for reprints should be addressed to: A. BALldabrigA, M.D., Children's Hospital of the "Seguridad Social," Paseo del Valle de Hebron, s/n, Barcelona, Spain.

32. Accepted for publication September 26, 1973. 